\title{
Hereditary sensory and autonomic neuropathy with deafness and global delay
}

INSERM

\section{Source}

INSERM. (1999). Orphanet: an online rare disease and orphan drug data base. Hereditary sensory and autonomic neuropathy with deafness and global delay. ORPHA:139573

This syndrome is characterized by a sensory and autonomic axonal neuropathy, sensorineural hearing loss and persistent global developmental delay. 